\title{
The effect of platelet-rich fibrin on the acceleration of osteoblast proliferation after endosseous dental implant insertion
}

\author{
Evan Chandra*,**, Suparyono Saleh" ${ }^{\star \star *}$, Murti Indrastuti***凹 \\ *Dr. Moewardi Hospital Surakarta, Central Java, Indonesia \\ **Prosthodontics Specialty Program, Faculty of Dentistry, Universitas Gadjah Mada, Yogyakarta, Indonesia \\ ***Department of Prosthodontics, Faculty of Dentistry, Universitas Gadjah Mada, Yogyakarta, Indonesia \\ *** JI Denta No 1, Sekip Utara, Yogyakarta, Indonesia; $₫$ correspondence: murtident@ugm.ac.id
}

Submitted: $3^{\text {rd }}$ December 2018; Revised: 20th June 2019; Accepted: $1^{\text {st }}$ November 2019

\begin{abstract}
The osseointegration process of dental implants begins with osteoblast differentiation and proliferation, which is an important aspect of the bone regeneration process. Platelet-rich fibrin (PRF) is a platelet that contains a large amount of fibrin and growth factor, which is widely used to accelerate bone regeneration. This study aims to determine the effect of PRF on the acceleration of osteoblast proliferation after endosseous dental implant insertion. Twelve male Wistar rats weighing 250 to $300 \mathrm{gr}$ of 8 to 15 weeks as an animal model were divided into two groups, the control group and treatment group, based on days and PRF treatment. Three $\mathrm{ml}$ of Wistar rat blood was obtained and centrifuged for 12 minutes at a speed of $2700 \mathrm{rpm}$ to make PRF. Afterward, platelet-rich fibrin was applied to implant bed, and the dental implant was inserted at the lateral epicondyle of the right femur in $3 \mathrm{~mm}$ depth and $1.8 \mathrm{~mm}$ diameter dimension. In control groups, dental implants were immediately inserted after implant bed preparation without PRF administration. Observation of the Wistar rats was carried out on days 14 and 28 for each group. The rats were terminated according to the timeline of group design. The epicondylus lateralis femoris dextra bone of the rats was taken and fixed with $10 \%$ buffered formalin solution. Then, histological samples were made with hematoxylin-eosin staining. Sample observation was done under a light microscope to calculate the number of osteoblasts. The data were analyzed using the two-way ANOVA test, followed by LSD post hoc test. Based on the two-way ANOVA test, there was a significant difference between control groups and treatment groups $(p<0.05)$, and there were significant differences between the observation result of days 14 and 28 ( $p<0.05)$. The application of PRF increased osteoblast proliferation for the first 14 days, but the rate decreased after that. Based on the LSD post hoc test, there were differences in osteoblast proliferation between the treatment and control group $(p<0.05)$. Thus, it is concluded that platelet-rich fibrin increased the acceleration of osteoblast proliferation after endosseous dental implant insertion.
\end{abstract}

Keywords: dental implant; growth factor; osteoblast; osseointegration; platelet-rich fibrin

\section{INTRODUCTION}

A dental implant is normally used to replace missing teeth and ideally to restore mastication, esthetic, phonetic function, and tooth-supporting tissue. A dental implant is placed by way of surgery into the jawbone as a replacement of roots to support prosthesis and dental bridges. ${ }^{1}$ The material of a dental implant is an important factor for osseointegration. Some materials, such as stainless steel, tantalum, and ceramic produce osseointegration, are widely used today. Titanium, however, remains the first choice of dental implant material because it is a reactive metal that spontaneously creates an oxide layer when exposed to air. This layer is waterproof and can hold against body fluid, can be formed into small parts, and thus can be implanted into the alveolar bone. In addition, this material is known to withstand the force of mastication to enable the bone growth around the surface of titanium. ${ }^{2}$

Osseointegration, as the main phenomenon in dental implants, is a form of attachment between bone tissues and dental implant surfaces embedded in the alveolar bone. Biomolecular events and bone cells play important roles in supporting osseointegration. During insertion of a dental 
implant, bone-to-implant contact $(\mathrm{BIC})$ is the main objective because this condition showed perfect osseointegration process, which resulted in a bond between substructure and surrounding alveolar bone without interference from fibrous tissues. ${ }^{3}$

Bone healing stages reflect the osseointegration stages. The osseointegration process begins from the insertion of a dental implant, bleeding, and acute inflammatory response during bone drilling, which is followed by a blood clot between implant and bone. Afterward, procallus is formed, and simultaneously, mesenchymal cells are differentiated into osteoblasts and fibroblasts. Osteoblasts then produce calcified fiber. Fibrocartilage, which is resulted from procallus, is developed into a woven bone in the third and fourth weeks. After seven weeks of post-implant insertion, the woven bone becomes lamellar bone. It is a more mineralized bone; therefore, it stabilizes the implant. Radiographically, the bones surrounding the dental implant in this stage are normal. The final stage of osseointegration can be seen from the maturation of bone surrounding the implant, increased density, and hardness. This stage continues until 1-year post-implant insertion. ${ }^{4}$

The surface of an implant plays an important role in the first stage of BIC. The formation of the bone surrounding the implant depends on bone healing ability. Thus, a chemically modified implant surface, such as a combination of organic substances to the titanium layer, has successfully increased the BIC level. Implant abutment is usually place after insertion at about 3-6 months for the mandible and 6-9 months for maxilla. It is necessary to consider the application of preparations that trigger bone regeneration and extend the osseointegration. There have been various methods to reduce the amount of time needed to reach osseointegration. ${ }^{5}$

Growth factors (GFs) are among the factors that induce osseointegration, and thus creating replication and differentiation of osteoprogenitors and tissue maturation. Osteoprogenitors are primitive mesenchymal cells that produce osteoblasts during bone growth and osteocytes in the layers inside bone tissues. ${ }^{6}$ In hematology, $\mathrm{PRF}$ is one of the growth factors available in human blood. PRF has been used widely for alveolar bone regeneration, sinus lifting procedure, and gingival recession closure in the form of membrane. ${ }^{7} \mathrm{PRF}$ is known to induce mitotic activity in osteoblasts, gingival fibroblasts, periodontal ligament cells, and suppress epithelial proliferation. PRF membrane inhibits early invagination of gingival epithelium, and thus it can act as a barrier for epithelial migration. ${ }^{8}$

During blood sample examination, PRF gives a lot of important elements of its use, a rich number of platelets, and cytokine leukocytes. Platelets in PRF contain a lot of growth factors, including platelet-derived growth factor (PDGF), transforming growth factor-beta (TGF- $\beta$ ), insulin growth factor (IGF), endothelial growth factor (EGF), fibroblast growth factor (FGF), and bone morphogenetic protein (BMP). These growth factors hold important roles in hemostasis, angiogenesis, proliferation, and differentiation of osteoblasts. ${ }^{9}$ This study aims to determine the effect of PRF to the acceleration of osteoblasts proliferation post endosseous dental implant insertion (in vivo study).

\section{MATERIALS AND METHODS}

This study is a laboratory experiment (in vivo) on 12 male Wistar rats of 8-15 weeks old with the average weight of 250-300 grams. The ethical approval of this study was obtained from ethical committee of Gajah Mada University, Faculty of Dentistry No. 001534/KKEP/FKG-UGM/EC/2018. Wistar rats were divided into treatment and control groups $(n=6)$ with 14 and 28-observation days for each group. This study used the endosseous implant (SD implant, C-TECH) of $1.8 \mathrm{~mm}$ diameter that was cut evenly at $3 \mathrm{~mm}$. The PRF used in this study was obtained from the blood of animal models that were centrifuged for 12 minutes at 2700 rpm speed using centrifugation machine (MPW-55, MPW med).

The right upper legs of Wistar rats were anesthetized intramuscularly using 1:1 xylazine and ketamine (each was $0.15 \mathrm{ml}$ ). In the treatment group, $2 \mathrm{ml}$ of blood was extracted from orbital sinus for PRF, and it was centrifuged for 12 minutes at $2700 \mathrm{rpm}$. The PRF membrane was taken from the second layer of centrifugation result. Fullthickness flap incision of $\pm 1 \mathrm{~cm}$ was performed on 
the right femoral lateral epicondyle. Bone drilling on the incised area was done using a pilot drill with $1.3 \mathrm{~mm}$ diameter and $2 \mathrm{~mm}$ depth, and $500 \mathrm{rpm}$ bur speed was performed to form implant bed. Treatment group was given $0.1 \mathrm{ml}$ of PRF into the implant socket, and the implant was placed into the socket at bone level. Meanwhile, in the control group, the implant was immediately placed after implant bed preparation. The incision wound was sutured to close the implant. Wistar rats were maintained according to the designed observation days, of 14 and 28 days. Afterward, the Wistar rats were terminated according to treatment group using dislocation method. The bone in the implant area was taken, and bone tissues were fixated using $10 \%$ buffered formalin and left for at least 24 hours. Bone tissues were cleaned from soft tissues along the surface of the bone. The bone was immersed in formic acid-HCL solution for 48 hours to soften the tissue. The implant was taken out carefully to avoid damage to the bone thread. Subsequently, the tissues were embedded in wax blocks, and cut using microtome to form preparations. The preparations were stained using hematoxylin-eosin then observed using a light microscope. 10x, 40x, and 100x magnifications were needed to observe the preparations. Magnification 10x was used to observe implant socket, $40 x$ to observe implant thread, and 100x to observe osteoblasts on the sides of the thread. Implant socket was divided into two, i.e. right and left thread sides, then observation and osteoblasts count were performed manually at 100x magnification. All osteoblasts in one thread were counted using a tally counter, and the count was repeated for 3 times and averaged.

The experiment resulted in ratio scale data, and thus parametric test was used. The data were tested using one-way ANOVA using significance level of $95 \%$. If the ANOVA test showed differences, the Post Hoc Least Significant Difference (LSD) test was performed to determine which group had significant differences.

\section{RESULTS}

The results of means and standard deviation of osteoblasts proliferation post-implant insertion in the femoral bone of Wistar rats that were given PRF in the drilling socket and implant insertion without PRF are presented in Table 1.

The data resulted in ratio scale and the scale was analyzed using two-way ANOVA parametric test. The two-way ANOVA test requires normally distributed data and homogenous variance. The normality test using Shapiro-Wilk showed $p>0.05$. It means that the data were normally distributed. The homogeneity test using Levene's test showed $p 0.852(p>0.05)$, therefore the data were homogenous. According to both tests, the data were eligible for parametric test. The result of twoway ANOVA parametric testis presented in Table 2.

The results of two-way ANOVA (Table 2) indicate that there was a significant difference of osteoblasts proliferation between the PRF group and control group $(p<0.05)$. Afterward, the LSD Post Hoc test was used to determine the group with significant differences. The Post Hoc test revealed that there was significant difference of osteoblasts proliferation between all treatment groups and observation days $(p<0.05)$. The results of LSD Post Hoc test can be seen in Table 3 .

Table 1. Means and standard deviation of osteoblasts proliferation with and without platelet-rich fibrin (PRF) on day 14 and 28

\begin{tabular}{lcc}
\hline Group & Control & PRF \\
\hline Day 14 & $52.33 \pm 5.82$ & $142.06 \pm 8.69$ \\
Day 28 & $65.95 \pm 5.74$ & $184.22 \pm 7.56$ \\
\hline
\end{tabular}

Table 2. The results of two-way ANOVA of osteoblasts proliferation

\begin{tabular}{lrl}
\hline Variable & \multicolumn{1}{c}{$\mathrm{F}$} & $\mathrm{p}$ value \\
\hline PRF & 1300.72 & $0.000^{*}$ \\
Day & 93.53 & $0.000^{*}$ \\
Group * day & 24.51 & $0.000^{*}$ \\
\hline
\end{tabular}

Table 3. The result of LSD Post Hoc test of osteoblasts proliferation

\begin{tabular}{lcccc}
\hline Group & $\mathrm{K}$ & $\mathrm{K}$ & $\mathrm{P}$ & $\mathrm{P}$ \\
& day 14 & day 28 & day 14 & day 28 \\
\hline K day 14 & - & & & \\
K day 28 & $0.003^{*}$ & - & & \\
P day 14 & $0.000^{*}$ & $0.000^{*}$ & - & \\
P day 28 & $0.000^{*}$ & $0.000^{*}$ & $0.000^{*}$ & - \\
\hline
\end{tabular}


Majalah Kedokteran Gigi Indonesia. April 2020; 6(1): 55- 59

ISSN 2460-0164 (print)

ISSN 2442-2576 (online)

\section{DISCUSSION}

This in vivo study aims to determine the effect of PRF to the acceleration of osteoblasts proliferation post endosseous dental implant insertion. The analysis of variance revealed the significance level of $p<0.05$. Therefore, the hypothesis of this study is accepted. In addition, it is known that the application of PRF can increase the acceleration of osteoblasts proliferation post endosseous dental implant insertion, and thus accelerating osseointegration. There were significant differences between control group and PRF group. The PRF application showed good results on osteoblasts proliferation because the growth factor (GF) in PRF triggered osteoblast growth. GFs that played an important role were PDGF, FGF, vascular endothelial growth factor (VEGF), and TGF- $\beta 1$. PDGF contained in PRF helped stimulate osteoblast proliferation to develop new cells. FGF in PRF accelerated osteoblast maturation, thus fastening the proliferation of new osteoblasts formed. TGF- $\beta 1$ is a protein-rich GF that has an important role in bone regeneration, epithelial cell growth, and osteoblast proliferation control. TGF- $\beta 1$ is also a strong agent in inhibiting the development of osteoclasts and bone resorption. Interleukin- $\beta 1$ (IL- $\beta 1$ ) in GF is one of the main keys of the differentiation and proliferation of osteoblasts. IL-6 GF is also a multifunction cytokine that activates and triggers osteoblasts proliferation. ${ }^{6}$

There was an interaction between observation times, i.e. between days 14 and days 28 of the PRF application to osteoblasts proliferation post endosseous implant insertion. Proliferation and differentiation of osteoblasts were derived from mesenchymal stem cells (MSC), which were controlled by BMP. In BMP, there was TGF- $\beta 1$, which played a role in osteoblasts proliferation. The PRF application in an implant socket, which contained a lot of growth factors, accelerated osteoblasts proliferation because TGF- $\beta 1$ was constituted in GF. This proliferation acceleration occurred as long as the active period of PRF in the socket. Therefore, after 14 days post-implant insertion, the PRF had started to resorb, and thus allowing the normal occurrence of osteoblasts proliferation.
The number of osteoblasts in PRF group on days 28 showed less acceleration than that of the first 14 days. This was not due to the fact that osteoblasts no longer formed, but was more attributed to the fact that osteoblasts had started to differentiate into osteocytes. Based on the process of bone formation, fibrocartilage started to form in the fourth week as the result of osteoblast and fiber in the osteointegration process. ${ }^{10}$

The LSD post hoc test showed significant differences in each group. The numbers of osteoblasts were higher in days 14 of PRF group compared to those of days 14 of control group. This showed the effectivity of PRF application in the socket for implant insertion. The number of osteoblasts in days 14 of PRF group was almost three times of the number of osteoblasts in days 14 of control group. The number of osteoblasts in days 28 of PRF group was less than that of the first 14 days. This result showed the effectiveness of PRF to increase in the first 14 days despite the gradual decrease after the remaining 14 days. This may be due to the fact that PRF acted as the GF-rich fibrin network, and thus allowing the gradual and controlled GF release. ${ }^{11}$

\section{CONCLUSION}

The application of PRF can accelerate osteoblasts proliferation post endosseous dental implant insertion.

\section{SUGGESTION}

To develop this topic, further studies need to use a specially designed dental implant for animals.

\section{REFERENCES}

1. Papaspyridakos P, Mokti M, Chen CJ, Benic GI, Gallucci GO, Chronopoulos V. Implant and prosthodontic survival rates with implant fixed complete dental prostheses in the edentulous mandible after at least 5 years: a systematic review. Clin Implant Dent and Relat Res. 2014; 16(5): 705-717. doi: 10.1111/cid.12036.

2. Parithimarkalaignan $S$, Padmanabhan TV. Osseointegration: An Update. J Indian 
Prosthodont Soc. 2013; 13(1): 2-6. doi: 10.1007/s13191-013-0252-z

3. Linkow LI, Wagner JR, Chanavaz M. Tripodal mandibular subperiosteal implant: basic sciences, operational procedure, and clinical data. J Oral Implantol. 1998; 24(1): 16-36. doi: 10.1563/1548-1336(1998)024<0016: TMSIBS>2.3.CO;2

4. Dover MS. Maxillofacial Surgery $2^{\text {nd }}$ Ed. Vol2 Chapter 81 Oseointegration. Churchil Livingstone: Elseiver; 2007.

5. Weiss C. Principles and Practice of implant dentistry 1st Ed. St Louis, New York: Mosby Inc; 2001. 6-14.

6. Oncu E, Bayram B, Kantarci A, Gulsever S Alaaddinoğlu EE. Positive effect of platelet rich fibrin on oseointegration. Med Oral Patol Oral Cir Bucal. 2016; 21(5): e601-7. doi: $10.4317 /$ medoral. 21026

7. Dohan DM, Choukroun J, Diss A, Dohan SL, Dohan AJ, Mouhyi J, Gogly B. Platelet rich fibrin(PRF): a second-generation platelet concentrate. Part I: Technological concept and evolution. Oral Surg Oral Med Oral Pathol Oral Radiol Endod. 2006; 101(3): e37-44.

doi: 10.1016/j.tripleo.2005.07.008
8. Tanya J, Thomas BS. Platelet rich fibrin membrane for recession coverage. EJ Dent. 2012; 2: 223-227.

9. Kökdere NN, Baykul T, Findik Y. The use of platelet-rich fibrin (PRF) and PRF-mixed particularedautogenous bone grafr in the treatment of bone defects: An experimental and histomorphometrical study. Dent Res J (Isfahan). 2015; 12(5): 418-424. doi: $10.4103 / 1735-3327.166188$

10. Dallas SL, Bonewald LF. Dynamics of the transition form osteoblast to osteocyte. Ann N Y Acad Sci. 2010; 1192: 437-443. doi: 10.1111/j.1749-6632.2009.05246.x

11. Dietrich $F$, Hammerman $M$, Blomgran P, Tätting L, Bampi VF, Silva JB, Aspenberg P. Effect of platelet-rich plasma on rat Achilles tendon healing is related to microbiota. Acta Orthop. 2017 Aug; 88(4): 416-421. doi: $10.1080 / 17453674.2017 .1293447$ 